\title{
EPIDEMIOLOGY
}

\section{Ethnicity, vitamin D, and CHD}

Low serum levels of 25-hydroxyvitamin D are associated with an increased risk of incident coronary heart disease (CHD) events in white or Chinese adults but not in black or Hispanic adults, new findings reveal.

An association between low serum levels of 25-hydroxyvitamin D and CHD has been found; however, most of the populations studied have included only or mainly white individuals. Evidence that this association exists in individuals of other ethnicities is lacking. "The MultiEthnic Study of Atherosclerosis (MESA) was an ideal place to examine whether associations of low 25-hydroxyvitamin D levels with CHD risk were consistent across ethnicity groups," says lead researcher Cassianne Robinson-Cohen of the Kidney Research Institute at the University of Washington, USA.

The researchers examined the association of 25-hydroxyvitamin D levels with CHD events among 6,436 participants of MESA who were free of cardiovascular disease at baseline.
The 25-hydroxyvitamin D levels measured at baseline were adjusted to account for seasonal variation in levels. Participants were followed up for a median of 8.5 years for the occurrence of CHD events, including myocardial infarction, angina, cardiac arrest, or death attributable to CHD.

Each $25 \mathrm{nmol} / 1$ decrement in levels of 25-hydroxyvitamin D was associated with an increased risk of CHD events in white (HR 1.26, 95\% CI 1.06-1.49) and Chinese (HR 1.67, 95\% CI 1.07-2.61) participants, after adjustment for known risk factors of $\mathrm{CHD}$; however, 25-hydroxyvitamin D levels were not associated with CHD risk in black or Hispanic participants. "Our results suggest that researchers should use caution when extrapolating results from studies conducted in white individuals to all ethnic groups," comments Robinson-Cohen.

The researchers speculate that biological differences explain most of the ethnic heterogeneity found in the effect of 25-hydroxyvitamin D levels on the

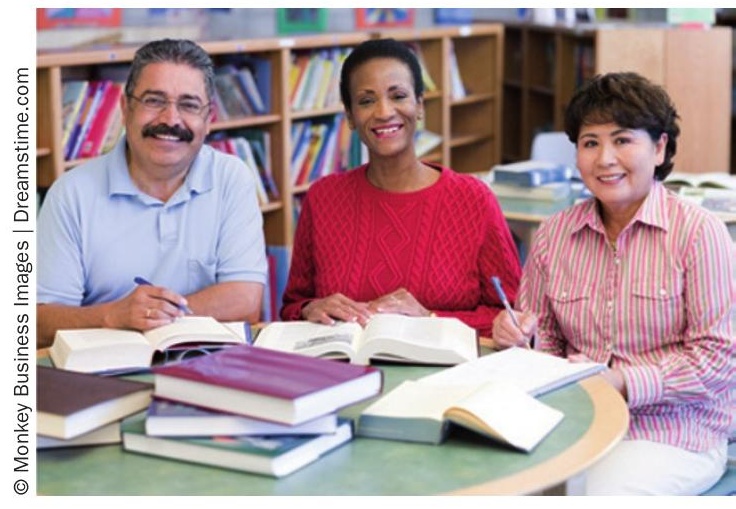

risk of CHD events. "We are currently planning to evaluate genetic variability in vitamin D metabolism and susceptibility to adverse effects of low levels of 25-hydroxyvitamin D across ethnic groups," comments Robinson-Cohen.

\section{Carol Wilson}

This article originally appeared in Nat. Rev. Endocrinol. (doi:10.1038/nrendo.2013.147).

Original article Robinson-Cohen, C. et al. Racial differences in the association of serum 25-hydroxyvitamin D concentration with coronary heart disease events. JAMA 310, 179-188 (2013) 